\title{
AToM: Atomic Topology Management of Wireless Sensor Networks
}

\author{
S. Shen ${ }^{1}$, G.M.P. O’Hare ${ }^{1}$, D. Marsh ${ }^{1}$, D. Diamond ${ }^{2}$, and D. O’Kane ${ }^{1}$ \\ ${ }^{1}$ Adaptive Information Cluster \\ School of Computer Science and Informatics \\ University College Dublin, Belfield, Dublin 4, Ireland \\ \{song.shen, gregory.ohare, david.marsh, donal.okane\}@ucd.ie \\ ${ }^{2}$ School of Chemical Sciences \\ Dublin City University, Dublin 9, Ireland
}

\begin{abstract}
This paper explores the structural and behavioural similarities that exist between the chemical constitution of natural substances and WSN (Wireless Sensor Network) topology. It introduces Atomic Topology Management (AToM), which uses concepts like WSN electron, WSN nucleus, WSN photon, and WSN atom to model sensor node, base station, message and basic WSN subset consisting of one base station and several sensor nodes. We subsequently extend the modeling to explain the basic behaviours of WSNs. The paper describes naming rules for topology management and based upon this, we devise and test some basic algorithms for energy and load balancing.
\end{abstract}

Keywords: Wireless Sensor Network, Topology Management, Autonomic Networking.

\section{Introduction}

Recent technology improvements in wireless communications and electronics have enabled the development of low-cost, low-power, multi-functional sensor nodes which possess the potential for self-organization and which can opportunistically collaborate in order that they may more effectively achieve, and continue to achieve, their overall goal. Large-scale sensor applications can be realized with such densely distributed micro nodes [1] [2].

Micro sensors of this nature are inherently resource-bounded, specifically with regard to energy (usually supplied by AA batteries). Such sensor nodes can typically merely communicate directly with those neighbours within a limited distance, rather than communicating with a Base Station (BS). A typical Wireless Sensor Network (WSN) consists of one or several BSs together with a large quantity of sensor nodes which sense the environment and work collaboratively, processing and routing the sensor data.

Little consideration has, as yet, been given to the issues of proper naming and topology management [13] [14] without which, management and analysis of the basic behaviors of a WSN are difficult. Several basic issues, such as the management of 
sensor network, coverage of sensor nodes [11], load balancing of neighbour subsets of WSN [3] [4] and routing for event data [8] [9] [10] [12], still remain open issues. These issues are closely related to self-configuring (or more generally topology management) of WSNs, since wireless communication is vulnerable to environmental changes. As sensor networks scale up in size, effectively identifying and managing the distribution of the network load will become of paramount importance. Creating a naming rule and building up a management model could well address and simplify research problems on architectural management and routing. However, modelling topology and the basic behaviours of sensor networks is inherently difficult because unlike other computer networks, the basic communication behaviours of the wireless sensor nodes can be described by a series of relayed discrete hops, each of which consumes approximately the same amount of energy. Within a WSN subset, a base station often serves as the data aggregation point or the sink of the data in the network, while those sensor nodes scattered around it are considered as resource limited relay points. With power consumption always the top priority, the basic behaviours of WSNs are fundamentally different from other networks.

Within this paper, we investigate possible analogues that may enable the more effective management of topologies of WSNs. We assume that the sensor network in question is not of a fixed nature determined in an a priori manner but rather one that comprises of sensors that are mobile and which potentially can determine when and where to move and/or respond to requests to do so. Therefore the naming and topology management of a WSN will be fundamental to its autonomic behaviours, such as self-configuring, self-healing, self-optimizing, and self-protecting. Fortunately, the world offers many resemblances to WSNs from which we can borrow. In particular, within this work we examine the chemical composition of natural substances. We advocate borrowing from this domain in the management of WSN topologies in this regard this work is pioneering and novel. Furthermore it is our contention that such a model can effectively underpin autonomic decision making and assist in the delivery of self properties. This analogy forms the core component of this paper which seeks to explore the appropriateness of the model in the WSN arena. The analogy helps us primarily in two aspects: one is guiding us to the naming and efficient management of a large topology of sensor networks in a natural way; the other is helping us to find a solution to energy and load balance, which can significantly improve network lifetime and communication ability.

\section{A Naming Convention Within Network Architectures}

\subsection{Definitions}

Considering the analogy with chemical compounds we now introduce some WSN definitions which embrace this viewpoint. Within the scope of this paper we consider chemical characteristics are beyond our concern. Therefore, we do not employ concepts such as isotope or ion in our approach.

The core definitions we adopt are now introduced:

Atom: An atom is composed of a nucleus, with electrons orbiting around it. A WSN atom, containing a base station and a subset of sensor nodes, is the smallest 
functional element within a sensor network just like a natural atom. Without losing generality, we assume that the WSN atom contains one BS.

Photon: A particle of light, associated with a piece of energy needed for an electron to jump to a higher energy level. In WSNs, a photon may be considered to equate to a message flow together with a unit of energy consumption associated with a single hop. The energy for a photon depends on the wavelength of the light; while in the WSN, the energy for a photon is approximately proportional to the length of the message.

Electron: A negatively charged particle orbiting around the nucleus. Within a WSN, an electron may be viewed as analogous to a sensor node distributed around a base station. A WSN electron is also a spot where each hopping message could temporarily reside.

Nucleus: The core of an atom. Within a WSN, the nucleus is viewed as the base station.

Molecule: A molecule, consisting of one or several atoms, is the smallest particle of a compound that has all the chemical properties of that compound. A WSN molecule, consisting of several WSN atoms, is a fundamental application unit of WSNs. A WSN molecule may consist of two or more different types of WSN atoms (Compound), see Fig. 1. The minimum WSN molecule is that of a single WSN atom.

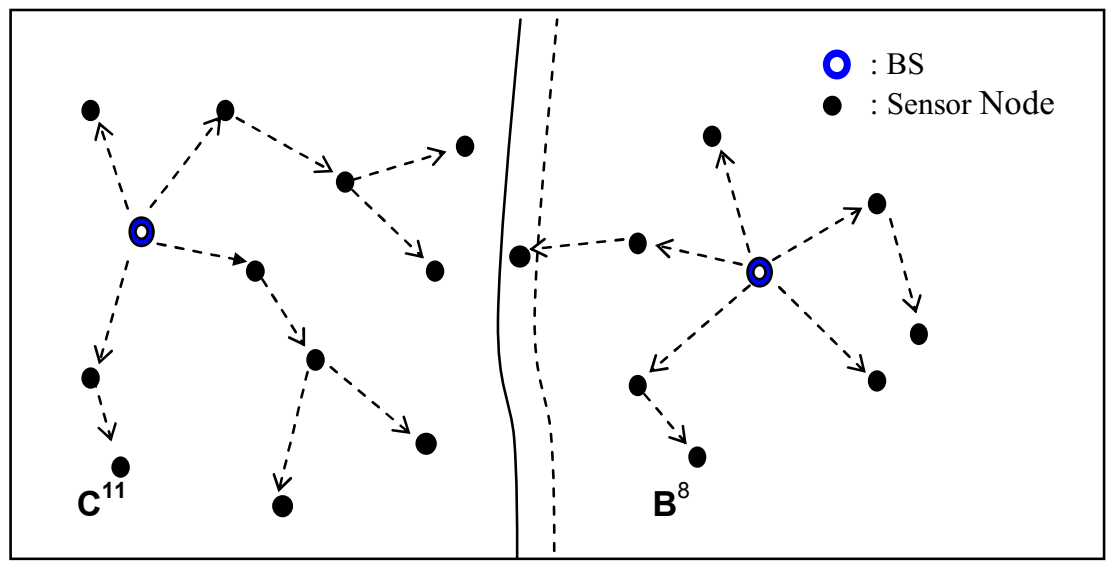

Fig. 1. WSN molecule example

\subsection{Similarities and Differences}

Based upon these definitions we now explore further the potential benefits of applying this analogy. The atom is an ultimately indivisible particle of an element in nature, while the WSN atom which we use to model a wireless sensor subset, is the smallest existing element of the WSN. On the other hand, a molecule is the smallest functional form of substance, while a WSN molecule, is a fundamental application unit of WSNs. In order to address the process of routing, we calibrate the distance from a given node to the BS into a number of hops, as shown in Fig. 2. Ideally, each hop should be able to move a piece of message onto another node closer to the BS. 
Clustering of WSN can be considered as a process of determining each sensor nodes' distance level to BS. The routing of messages toward the BS within a WSN is a process of hopping to a higher level in discrete steps, although the levels may not necessarily be well defined beforehand. Such a process is inherently similar to the jumping of electrons on a higher energy level within atomic structures. The major similarities can be concluded as:

First of all, data from multiple sensor nodes are all required to pass to a particular $\mathrm{BS}$ or a few BSs. The BS can be regarded as the centre of a group of nodes, just like the nucleus of an atom. A certain number of sensor nodes together with a BS make a basic unit of WSN subset, which is the smallest usable WSN element, or a WSN atom. The number of sensor nodes within the WSN element together with their energy levels determines characteristics of the WSN element, just as chemical characteristics of an atom is decided by its position on the Periodic Table of Elements. Furthermore, necessities to compose WSN subsets and to balance load on sensor nodes for each WSN subsets or for each level within a subset expedite some kind of distribution rules for sensor nodes. Such composing and balancing behaviours could be modelled by chemical reactions.

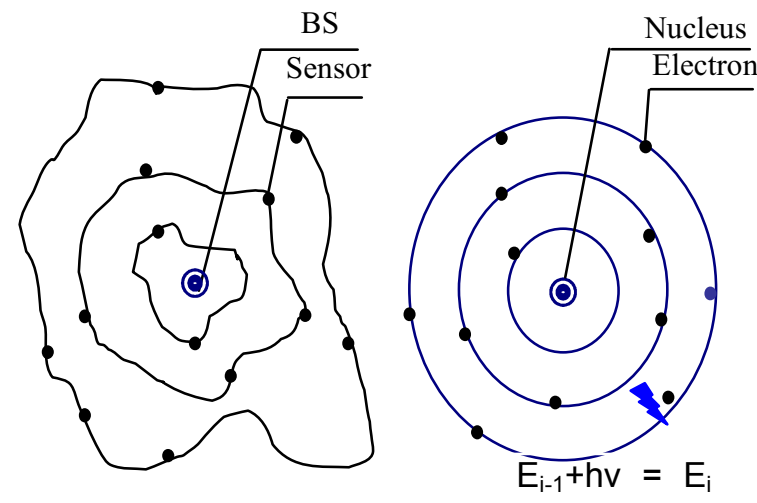

Fig. 2. Modelling of levelled sensor nodes

Second, several different WSN elements can combine into a hybrid structure, like a chemical molecule can be composed of several different chemical elements. The naming convention for such reactions or derived compounds could borrow from that used in chemical reactions.

Third, akin to the jumping of an electron to a higher energy level, each hop of message within a WSN consumes a piece of energy. The energy cost of hopping a message to a higher level is approximately proportional to the length of the message, while energy cost of an electron jump is proportional to the wavelength of the emitted photon. Both are based on the concept of energy level and are independent of the distance of physical movement.

There are also many differences between an atom and a WSN. For example, the constitution of a WSN can have as many levels as necessary and the number of sensor 
nodes on each level does not have to strictly follow a rule similar to Pauli's Exclusion Principle.

One major difference emerges from the fact that although we simulate sensor nodes as electrons in a WSN subset, they are normally static geographically. What actually hops is the message, rather than sensor nodes themselves. However, our primary concern is clustering related characteristics such as coverage of sensor nodes, the load balancing of neighbouring subsets of WSN, as well as the energy related characteristics such as consumption of message hopping. Therefore, the jumping characteristics of electrons can merely be considered as the flow of a message. In fact, it is not necessary for a WSN to adopt all the characteristics synonymous with the natural structure.

\subsection{Naming of WSN Atom, WSN Molecule and WSN Electron}

A WSN compound needs to know the structure, constitution and level situation of its WSN atoms for management purposes. Therefore, proper naming of sub items within a WSN is crucial. With the basic terms defined in the preceding section, we can further visually name the structure of WSN atoms and WSN molecules. Such naming could be flexible according to the scale of the WSN and the elaboration necessities. For example, we could use A to denote a WSN atom with only one external level; use B to denote two external levels, $\mathrm{C}$ to denote three external levels, and so on. If so, a WSN subset with one BS and 11 sensor nodes distributed in 3 levels could be denoted as $\mathrm{C}^{11}$; a WSN subset with one BS and 8 sensor nodes distributed in 2 levels could be denoted as $\mathrm{B}^{8}$; a WSN molecule composed of WSN atoms $B^{8}$ and $C^{11}$ is named as $C^{11} B^{8}$, while a WSN electron within WSN atom $C^{11}$ can be named as $C^{11}, i, j$, where $i$ is the level where the electron situates at and $\mathrm{j}$ is the sequence number of the electron, see Fig. 1.

Furthermore, if certain sensor placement principles could be formalized for determining the placement of sensor nodes within a given level, it would be possible to define a table of WSN elements, similar to the Periodic Table of Chemical Elements.

\section{Behaviours of WSNs}

\subsection{Basic Behaviours}

\subsubsection{Composing a WSN Atom}

WSN atoms are formed during the period of clustering. In this period, each sensor node is registered to a neighbouring BS.

\subsubsection{Composing a WSN Molecule}

Several WSN atoms (or sometimes only one WSN atom) form a WSN molecule. Like covalent bonds, in some occasions a WSN electron can work for more than one WSN atoms at the same time. Analogous to naming of chemical molecules, the WSN molecule is named after each concerned WSN atom. For example, the molecule demonstrated in Fig. 1 is named as $\mathrm{C}^{11} \mathrm{~B}^{8}$.

\subsubsection{Adding a WSN Electron}

Whenever a new node is added into an atom, it will connect to its neighbours and decide its own level within the network. The addition of a new WSN electron will 
change the structure and the naming of the WSN atom. In Fig. 1, for example, as the boundary of the two WSN atom changes to the dashed line, the left atom will become $\mathrm{C}^{12}$. If the new electron can only connect to the BS through some of the outmost electrons, then the addition will also change the number of layers.

\subsubsection{Subtracting a WSN Electron}

The more layers an atom has, the more active the external electrons. Outermost electrons may escape either at the attraction of another atom or by some other external force. Similarly, subtracting a WSN electron might occur when some WSN electrons escape away from their current WSN atoms to another less layered WSN atom as a result of some load balancing process or due to energy exhaustion. As shown in Fig. 1, when a WSN electron escapes away from the right WSN atom, the WSN atom becomes $\mathrm{B}^{7}$.

\subsection{Message Hopping}

Within the AToM model, we consider a WSN photon as analogous to message exchange with an associated energy consumption. The energy consumption is approximately proportional to the length of the message. A WSN electron, once accepting a WSN photon, will turn to an excited state which persists until the electron hops the photon to a neighbour. By releasing the photon, the electron will return to a ground state. This process will iterate until the photon reaches the BS.

The whole routing action can be expressed as $\left\langle\mathrm{H}_{1}, \mathrm{H}_{2}, \mathrm{H}_{3}, \ldots, \mathrm{H}_{\mathrm{N}}\right\rangle$, where each component hopping action $\mathrm{H}_{\mathrm{I}}$ is carried out at node I. Suppose the environment at node $\mathrm{I}$ is $\mathrm{e}_{\mathrm{I}}$ at some time point, the process is further denoted as a sequence of interleaved environment states $\mathrm{E}=\left\{e_{0}, e_{1}, \ldots e_{N}\right\}$ and actions $\mathrm{e}_{0} \stackrel{\mathrm{H}_{1}}{\longrightarrow} \mathrm{e}_{1} \stackrel{\mathrm{H}_{2}}{\longrightarrow} \mathrm{e}_{2} \stackrel{\mathrm{H}_{3}}{\longrightarrow} \underset{\mathrm{H}}{\longrightarrow} \stackrel{\mathrm{H}_{\mathrm{N}}-1}{\longrightarrow} \mathrm{e}_{\mathrm{N}}$.

See: $\mathrm{E} \rightarrow$ Per

Act: I $\rightarrow$ Hop

Next: I $\times$ Per $\rightarrow$ I

Fig. 3. Coordinated routing functions

Because of resource limitations, particularly on communication ability, the bottleneck of routing is predominantly at the sensor nodes' side. The coordinated hopping behaviours of sensor nodes are listed in Fig. 3 as three major functions. A see function maps the environment states to perception; an action-selection function act maps from internal states to hopping actions; a next function maps internal state and perception to an new internal state. These functions are executed recursively until the message finally reaches the BS. 
We adopt the simple assumption as described in [12], where the radio dissipates $\mathrm{Ee}=50 \mathrm{~nJ} / \mathrm{bit}$ to run the transmitter or receiver circuitry and $\mathrm{Ea}=100 \mathrm{pJ} / \mathrm{bit} / \mathrm{m}^{2}$ to run the transmit amplifier. Therefore, to transmit an m-bit message a distance $d$ expends

$$
\begin{aligned}
\mathrm{Et} & =\mathrm{Ee} \cdot \mathrm{m}+\mathrm{Ea} \cdot \mathrm{m} \cdot \mathrm{d}^{2} \\
& =\mathrm{m} \cdot\left(50+0.1 \cdot \mathrm{d}^{2}\right)(\mathrm{nJ})
\end{aligned}
$$

To receive this message, the radio expends

$$
\begin{aligned}
\mathrm{Er} & =\mathrm{Ee} \cdot \mathrm{m} \\
& =50 \cdot \mathrm{m}(\mathrm{nJ})
\end{aligned}
$$

If a message is sent through $\mathrm{H}$ hops and finally reaches the $\mathrm{BS}$, then the hopping energy expense $\mathrm{E}_{\mathrm{H}}$ is roughly expressed as

$$
\mathrm{E}_{\mathrm{H}}=\mathrm{H} \cdot \mathrm{Et}+(\mathrm{H}-1) \cdot \mathrm{Er}
$$

\section{Management of Energy and Load Balancing}

Having introduced the Atom naming conventions identified within Section 2 and the modelling of their behaviours presented within Section 3, we now turn our attention to the usage of such/ Specifically we now explore how this model could form the foundation of effective energy balancing amongst WSN electrons and load balancing among WSN subsets.

\subsection{Load Balancing Between WSN Atom Levels}

Assume that a WSN subnet contains one BS and N sensor nodes distributed into L levels, then the minimum energy consumption (hops at same levels are neglected) on each level for an operation of hopping a piece of a message from each node to the BS can be derived from Table 1.

Within a WSN, the restriction of sensor distribution on each hopping level is much looser than the distribution of electrons on different energy levels which is strictly defined by Pauli's law. However, the number of levels and the distribution of nodes on each level do make a difference to both energy efficiency and hopping cost within a given network. Energy balance is one of the major considerations in creating balance among levels, because it determines the life expectancy of the network. It can be seen from Table 1 that within a peer-to-peer network, sensor nodes closer to the BS have a heavier burden than those located at outer levels, if their hopping levels remain static. Consequently, the greater the number of nodes (within a fixed network) on low levels, the better the balance of the levels. Conversely the less the number of levels, the better the load balance of the network, assuming of course, no specific clustering techniques are applied. In addition, the fewer the number of levels the better the balance of power consumption among levels. 
Table 1. Energy consumption of routing on each level for a round of data collection

\begin{tabular}{|l|l|l|}
\hline Level & No. of nodes & $\begin{array}{c}\text { Minimum Energy consumption } \\
\text { on level }\end{array}$ \\
\hline $0(B S)$ & 1 & External energy supply \\
\hline 1 & $\mathrm{~N}_{1}$ & $\mathrm{~N} \cdot \mathrm{Et}+\left(\mathrm{N}-\mathrm{N}_{1}\right) \cdot \mathrm{Er}$ \\
\hline 2 & $\mathrm{~N}_{2}$ & $\left(\mathrm{~N}-\mathrm{N}_{1}\right) \cdot \mathrm{Et}+\left(\mathrm{N}-\mathrm{N}_{1}-\mathrm{N}_{2}\right) \cdot \mathrm{Er}$ \\
\hline 3 & $\mathrm{~N}_{3}$ & $\left(\mathrm{~N}-\mathrm{N}_{1}-\mathrm{N}_{2}\right) \cdot \mathrm{Et}+\left(\mathrm{N}-\mathrm{N}_{1}-\mathrm{N}_{2}-\mathrm{N}_{3}\right) \cdot \mathrm{Er}$ \\
\hline$\ldots$ & $\ldots$ & $\ldots$ \\
\hline $\mathrm{K}$ & $\mathrm{N}_{\mathrm{k}}$ & $\left(\mathrm{N}-\sum_{\mathrm{J}=1}^{\mathrm{K}-1}\left(\mathrm{~N}_{\mathrm{J}}\right)\right) \cdot \mathrm{Et}+\left(\mathrm{N}-\sum_{\mathrm{J}=1}^{\mathrm{K}}\left(\mathrm{N}_{\mathrm{J}}\right)\right) \cdot \mathrm{Er}$ \\
\hline$\ldots$ & $\ldots$ & $\ldots$ \\
\hline $\mathrm{L}$ (Last) & $\mathrm{N}_{\mathrm{L}}$ & $\mathrm{N}_{\mathrm{L}} \cdot \mathrm{Et}$ \\
\hline & \multicolumn{2}{|l}{} \\
\hline
\end{tabular}

\subsection{Management of Load Balance Among WSN Atoms}

We denote load balance level by an index called load imbalance level $\mathrm{B}_{\mathrm{L}}$, which is defined as the average unevenness of the corresponding numbers of WSN electrons of a group of $\mathrm{N}$ neighbouring WSN atoms $\left\{\right.$ atom $_{1}$, atom $_{2}, \ldots$, atom $\left._{\mathrm{N}}\right\}$.

$$
\mathrm{B}_{\mathrm{L}}=\left(\sum_{\mathrm{i}=1}^{\mathrm{N}}\left|\operatorname{Load}\left(\operatorname{atom}_{\mathrm{i}}\right) / \operatorname{Load}_{0}-1\right|\right) / \mathrm{N}
$$

Where $\operatorname{Load}\left(\right.$ atom $\left._{\mathrm{i}}\right)$ is a function to derive the number of WSN electrons of atom ${ }_{\mathrm{i}}$, $\operatorname{Load}_{0}$ is the average number of WSN electrons of the group. The greater the $\mathrm{B}_{\mathrm{L}}$, the lower the load balance level.

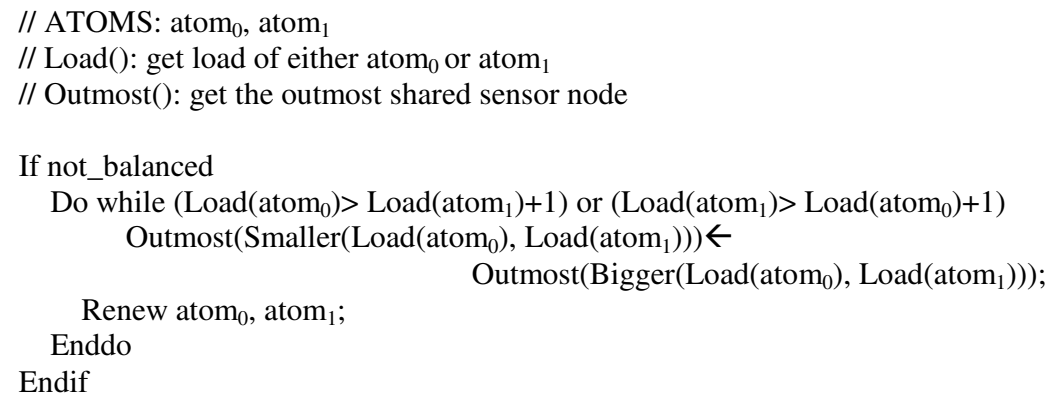

Fig. 4. Load balancing algorithm between WSN atoms

Load balancing among WSN atoms, the process of lessening the unevenness of their electrons, is an important step toward prolonged lifetime and efficiency for the whole network. The balancing between two neighbouring WSN atoms could be achieved with the algorithm presented in Fig. 4, by moving one or more sensor nodes from a larger atom to a smaller atom. It is important to note that we assume that sensors within the network have a mobility capability and can either decide or be advised 
to migrate to a new destination. Such migration is achieved by aggregated hopping with sensors always binding to a given base station in a manner similar to electrons. Balancing of three or more WSN atoms can be achieved based upon repeated balancing between two neighbouring WSN atoms.

\subsection{Management of Energy Balance Among Electrons on the Same Level}

We denote energy balance level by energy imbalance level $\mathrm{B}_{\mathrm{E}}$, which is defined as the average unevenness of the corresponding energy level of a group of $\mathrm{M}$ neighbouring WSN electrons $\left\{\right.$ electrons $_{1}$, electrons $_{2}, \ldots$, electrons $\left._{N}\right\}$.

$$
\mathrm{B}_{\mathrm{E}}=\left(\sum_{\mathrm{i}=1}^{\mathrm{M}} \mid \text { Energy }\left(\text { Electron }_{\mathrm{i}}\right) / \text { Energy }_{0}-1 \mid\right) / \mathrm{M}
$$

Where Energy $\left(\right.$ Electron $\left._{\mathrm{i}}\right)$ is a function to derive the energy level of electron $\mathrm{i}_{\mathrm{i}}$, while Energy $_{0}$ is the average energy of the group $\left\{\right.$ electrons $_{1}$, electrons ${ }_{2}, \ldots$, electrons $\left._{\mathrm{M}}\right\}$. The greater the $\mathrm{B}_{\mathrm{E}}$, the lower the energy balance level.

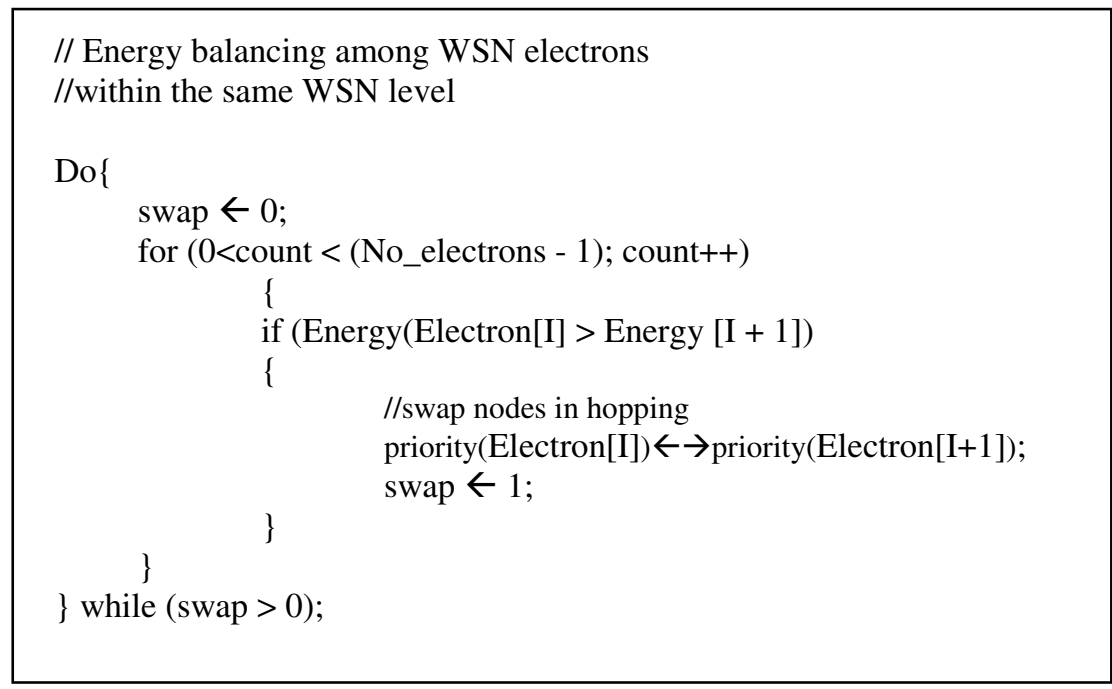

Fig. 5. Algorithm for energy balancing among WSN electrons

In order to prolong life expectancy of a WSN network, energy balance among WSN same-level electrons should be embedded into the management software. Many routing protocols support energy balance [6] [8] [9] [10] [12]. Among them, LEACH [12] is a clustering-based protocol that utilizes randomized rotation of cluster-heads to evenly distribute the energy load among the sensor nodes in the network. Within the management software, such rotation manifests itself as a swapping operation. Fig. 5 demonstrates the algorithm we adopt for energy balancing among electrons on the same level. 


\section{Case Studies}

A simulation was carried out on a randomly distributed sensor networks, where the above-mentioned balancing management was implemented, as demonstrated in the following case studies.

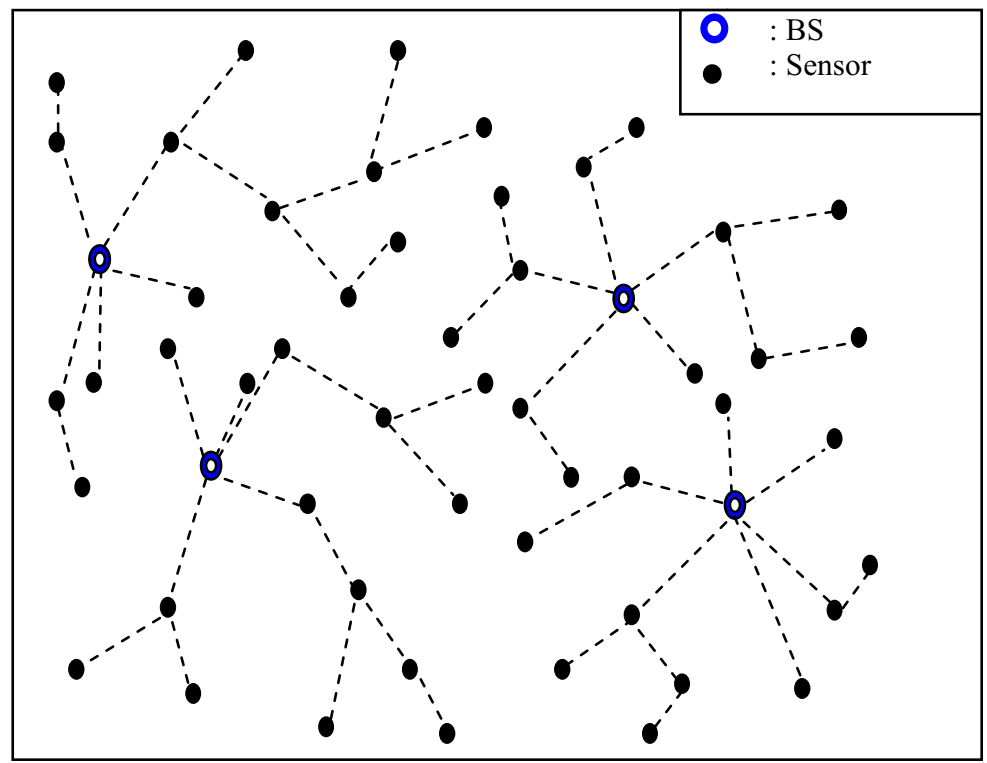

Fig. 6. Clustering and balancing of WSN atoms

The first case study deals with the clustering of a WSN consisting of 4 WSN nucleus and 51 WSN electrons where the threshold of the load imbalance level is set to $20 \%$. These sensor nodes were clustered dynamically according to their neighbours. The execution of the clustering algorithm results in a balanced structure of WSN atoms, $\mathrm{D}^{14}, \mathrm{C}^{12}, \mathrm{D}^{14}$, and $\mathrm{C}^{11}$, as a topological compromise shown in Fig. 6. From Eq.(4), the load imbalance level $\mathrm{B}_{\mathrm{L}}$ of this specific case is $12 \%$. This represents an $8 \%$ improvement with a reduced load imbalance level (see Section 4.2).

A second case study dealt with the energy balance of a WSN atom, $\mathrm{C}^{11}$, over a period of time. As demonstrated in Fig. 7, the white curve is the initial energy distribution of the 11 WSN electrons, while the black one is the energy distribution of these nodes some time later. It can be calculated from Eq. (5) that their initial energy distribution $\{0.98,0.78,0.71,0.78,0.6,0.68,0.74,0.91,0.78,0.66,0.82\}$ has an imbalance level of about $10.5 \%$. The energy-balancing algorithm improves the energy distribution to the black curve $\{0.59,54,0.5,0.53,0.45,0.48,0.51,0.56,0.52,0.5$, $0.54\}$ which has an energy imbalance level of about $5.6 \%$. The results demonstrate that through the execution of the energy-balancing algorithm, the energy imbalance level among these WSN electrons is improved by $4.9 \%$. 


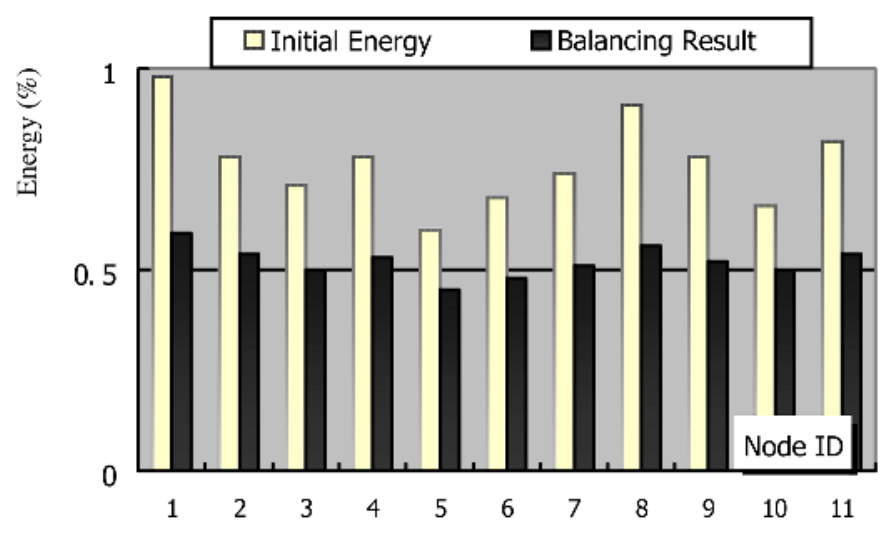

Fig. 7. Schematic view of energy balance

\section{Conclusions}

The Atomic Topology Management introduced in this paper is novel in its adoption of chemical structures and chemical reactions as a metaphor for WSN management. This is the first work that has advocated drawing such parallels as an instrument to intelligent management of WSNs and thus imbuing autonomic properties to such networks. This approach can be applied to the design and management of sensor networks on the MAC layer and routing layer. More specifically, it helps in evaluating WSNs' energy efficiency of routing, scalability, network lifetime, coverage and load balancing. The algorithms described within this paper have already been utilized within mote-based WSNs.

On-going and future research will focus on the completion of the management platform. In particular we are further extending the chemical analogy by investigating how to characterise not individual base station and sensor subnets, but rather the aggregation of numerous such subnets. We examine issues of overall stability of the compound/network and consider how this effects or inhibits reactions. We examine the Subnet Periodic Table as a classification mechanism for subnet structures. This borrows heavily from its chemical counterpart. We are exploring the concept of groups and periods and other classifications. Additional work considers subnet composition theories that will obviously stand in contrast to the Aufbau principle. Our research also examines such issues as chemical reactions caused between subnet components together with the possibility of co-valence bonds and how these could facilitate network adaptivity.

While Atomic Topology Management of Wireless Sensor Networks is a work in progress the efficacy of the model has already been demonstrated in effective naming and energy and load balancing algorithms. While these are rudimentary at this stage they demonstrate how the model can underpin autonomic WSN management and adaptivity delivering significant performance improvements. 


\section{Acknowledgements}

S. Shen, G.M.P. O'Hare, D. Marsh, D. Diamond, and D. O'Kane gratefully acknowledge the support of Science Foundation Ireland under Grant No. 03/IN.3/1361.

\section{References}

1. J. Hill, R. Szewcyzk, A. Woo, S. Hollar, D. Culler, K. Pister, "System Architecture Diretions for Networked Sensors", Proceedings of Ninth International Conference on Architectural Support for Programming Languages and Operating Systems, November 2000.

2. I. Akyildiz, W. Su, Y. Sankarasubramaniam, and E.Cayirci. "Wireless Sensor Networks: A Survey", Computer Networks, 38(4): 393-422, March 2002.

3. H. Dai, R. Han, "A Node-Centric Load Balancing Algorithm for Wireless Sensor Networks", IEEE GLOBECOM - Wireless Communications, 2003

4. P. H. Hsiao, A. Hwang, H. T. Kung, and D. Vlah. "Load-Balancing Routing for Wireless Access Networks", IEEE Infocom, April 2001.

5. Q. Li, J. Aslam, and D. Rus. "Hierarchical power-aware routing in sensor networks". In Proceedings of the DIMACS Workshop on Pervasive Networking, May 2001.

6. A. Manjeshwar and D. P. Agrawal. "Teen: A routing protocol for enhanced efficiency in wireless sensor networks". In 1st International Workshop on Parallel and Distributed Computing Issues in Wireless, 2001.

7. J. Zhao, R. Govindan and D. Estrin, "Residual Energy Scans for Monitoring Wireless Sensor Networks", IEEE Wireless Communications and Networking Conference (WCNC'02), Orange County Convention Center, Orlando, FL, USA, 17-21 March, 2002.

8. C. Intanagonwiwat, R. Govindan, and D. Estrin, "Directed diffusion: A scalable and robust communication paradigm for sensor networks", In the Sixth Annual International Conference on Mobile Computing and Networking, Boston, MA, USA, August 2000.

9. Y. Yu, R. Govindan, and D. Estrin, "Geographical and energy aware routing: a recursive data dissemination protocol for wireless sensor networks", Technical Report UCLACSDTR-01-0023, Computer Science Department, University of California at Los Angeles, May 2001.

10. A. Woo, T. Tong, and D. Culler, "Taming the underlying challenges for reliable multi-hop routing in sensor networks", SenSys'03, Log Angeles, CA, USA, November 2003.

11. X. Wang, G. Xing, Y. Zhang, C. Lu, R. Pless, and C. Gill, "Integrated Coverage and Connectivity Configuration in Wireless Sensor Networks", First ACM Conference on Embedded Networked Sensor Systems (SenSys'03), Los Angeles, CA, November 2003.

12. W. Rabiner, Heinzelman, A. Chandrakasan and Hari Balakrishnan, "Energy-Efficient Communication Protocol for Wireless Micro-sensor Networks", In Proceedings of the 33rd International Conference on System Sciences (HICSS '00), 2000.

13. J. Pan etc, Topology Control for Wireless Sensor Network, International Conference on Mobile Computing and Networking, Proceedings of the 9th annual international conference on Mobile computing and networking, San Diego, CA, USA, PP. 286 - 299, 2003.

14. A. Salhieh, J. Weinmann, M. Kochhal, and L. Schwiebert, Power Efficient Topologies for Wireless Sensor Networks, in International Conference on Parallel Processing, pages 156--163, Sept. 2001. 